This item was submitted to Loughborough's Research Repository by the author.

Items in Figshare are protected by copyright, with all rights reserved, unless otherwise indicated.

\title{
Large eddy simulation of crossflow vortices on an infinite swept wing
}

PLEASE CITE THE PUBLISHED VERSION

PUBLISHER

Published by the American Institute of Aeronautics and Astronautics, Inc. @ the author(s)

VERSION

VoR (Version of Record)

LICENCE

CC BY-NC-ND 4.0

REPOSITORY RECORD

Mistry, Vinan I., Gary J. Page, and James J. McGuirk. 2012. "Large Eddy Simulation of Crossflow Vortices on an Infinite Swept Wing”. figshare. https://hdl.handle.net/2134/10270. 
This item was submitted to Loughborough's Institutional Repository (https://dspace.lboro.ac.uk/) by the author and is made available under the following Creative Commons Licence conditions.

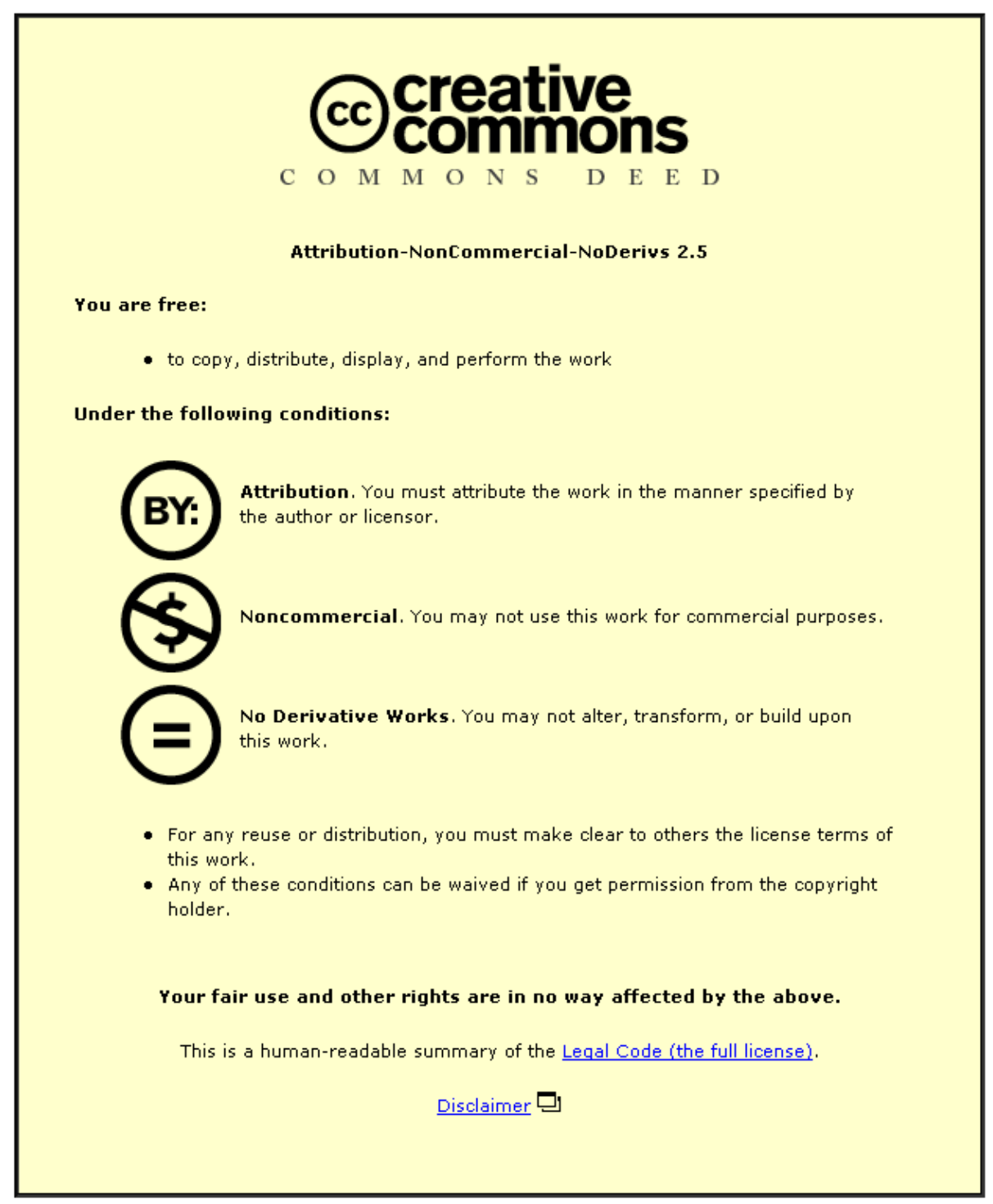

For the full text of this licence, please go to: http://creativecommons.org/licenses/by-nc-nd/2.5/ 


\title{
Large Eddy Simulation of Crossflow Vortices on an Infinite Swept Wing
}

\author{
Vinan I. Mistry* , Gary J. Page ${ }^{\dagger}$ and Jim J. McGuirk ${ }^{\ddagger}$ \\ Loughborough University, Loughborough, Leicestershire, LE11 3TU, UK
}

\begin{abstract}
Large Eddy Simulation (LES) was used to model the formation of a crossflow vortex packet in a 3-dimensional swept-wing boundary layer. The capability of LES to model the fine structures near the wall was investigated. An experiment by Chernoray et al. ${ }^{1}$ was used as a base case and the solution domain replicated their experimental setup with a C-16 airfoil at sweep $45^{\circ}$ and $R e_{c}=390,000$. Two sub-grid models were used for the investigation: the standard Smagorinsky and the Wall-Adapting Eddy Viscosity (WALE) model. The WALE model is more suitable as it allows the sub-grid scale viscosity to vanish in laminar regions and in the inner regions of the boundary layer. Stagnation streamlines at airfoil leading and trailing edges were taken from a full C-16 grid and used to define the lower boundary of a smaller solution domain which included the wing upper surface. This allowed, for a given computational resource, additional refinement in the area of interest. Results from the full grid matched well with that of the streamline defined domain. The laminar base flow for two LES grids of size 38 million \& 161 million nodes was compared against the experiment and the results agreed well although the LES results slightly over-predicted the boundary layer thickness compared to the experiment. Stationary crossflow vortices were generated by strong continuous suction through a $1 \mathrm{~mm}$ hole. The LES successfully captured the generation and growth of the crossflow vortex packet as well as the breakdown to turbulence on both grids. The fine grid performed better in modelling the growth of the vortices and the location of onset and growth of a dominant ' $z$ ' mode secondary instability. It was concluded that with suitable grid resolution LES is capable of successfully capturing the onset and development of crossflow vortices at a lower computational cost compared to DNS.
\end{abstract}

\section{Nomenclature}

$\begin{array}{ll}\mathrm{NO}_{X} & \text { Nitrous Oxides } \\ \mathrm{TS} & \text { Tollmien-Schlichting } \\ \mathrm{CFL} & \text { Courant Friedrichs Lewy Number } \\ \Delta & \text { Grid Filter } \\ \nu_{t} & \text { Eddy Viscosity } \\ \nu & \text { Kinematic Viscosity } \\ \mathrm{C}_{s} & \text { Smagorinsky Constant } \\ S_{i j} & \text { Strain-Rate Tensor } \\ \mathrm{C}_{w} & \text { WALE Constant } \\ \mathrm{Q}_{0} & \text { Freestream Velocity } \\ \mathrm{Q}_{e} & \text { Boundary Layer Edge Velocity } \\ \mathrm{Re}_{c} & \text { Chord Reynolds Number } \\ \mathrm{Re}_{\theta} & \text { Momentum Thickness Reynolds Number } \\ \mathrm{C}_{p} & \text { Pressure Coefficient } \\ \mathrm{C}_{f} & \text { Skin Friction Coefficient } \\ \mathrm{U}, & \text { Streamwise Disturbance Velocity }\end{array}$

\footnotetext{
*Research Engineer, Email: V.I.Mistry@lboro.ac.uk

†Senior Lecturer, Email: G.J.Page@lboro.ac.uk

$¥$ Professor of Aerodynamics, J.J.McGuirk@lboro.ac.uk
} 


\section{Introduction}

$T^{\text {HE }}$ aircraft industry has made significant progress in reducing its impact on the environment with aircraft 1 more than $70 \%$ more fuel efficient over the past 50 years. However, according to the Airbus Global Market Forecast $2011^{2}$ overall world passenger traffic is expected to increase $4.7 \%$ per annum in the next decade; for this reason the aircraft industry has had to research further into emissions reduction to provide a step change in aircraft efficiency for future aircraft. The industry has set ambitious targets in reducing $75 \%$ of carbon emissions from commercial aircraft, by 2050 , relative to 2000 levels. ${ }^{3,4}$ A large part of the reduction will be achieved by innovative new engine configurations and the increased use of advanced materials, ${ }^{5}$ however the expected benefits of these technologies will not be sufficient; in addition, reduction of aircraft drag using technologies such as laminar flow will be required to reduce the fuel burn and carbon emissions of future aircraft. Promoting laminar flow within a boundary layer has the potential for large savings in skin friction drag on aircraft. According to Schrauf ${ }^{5}$ if the boundary layer flow on a wing was laminar on $20 \%, 30 \%$ or $40 \%$ of the surface area, the total drag of the aircraft could potentially be lowered by $8 \%, 12 \%$ or $16 \%$, a significant reduction.

Laminar flow is achieved on a civil aircraft wing by shifting the onset of transition from laminar to turbulent flow within the boundary layer as far aft as possible. This is done by manipulating the instabilities that cause the onset of transition. Three main instability mechanisms generally contribute to transition on a swept civil aircraft wing. The first is due to a combination of leading-edge radius and sweep which leads to the attachment-line mechanism; control of this instability is achieved by adjusting the attachment line Reynolds number $(\bar{R}<245){ }^{6,7}$ The second are streamwise instabilities that occur in the form of the familiar Tollmien-Schlichting (TS) waves that grow exponentially and lead to transition in the mid-chord region. It is now well known that shaping the airfoil for a favourable gradient and minimising the extent of the pressurerecovery region both contribute to the control of this instability. In regions of pressure gradient on highly swept surfaces the third mechanism, crossflow instability, causes transition close to the leading edge. The combined influences of sweep and pressure gradient produce curved streamlines at the boundary-layer edge causing the streamwise velocity to reduce. However, the pressure gradient remains unchanged and thus the balance between centripetal acceleration and pressure gradient no longer exists, resulting in a secondary flow in the boundary layer, called crossflow. Because the crossflow velocity must vanish at the wall and at the edge of the boundary layer, an inflection point exists and provides the source of an inviscid instability. ${ }^{8}$ The crossflow instability exhibits amplified disturbances that can be stationary or travelling, with stationary vortical disturbances having been found to be more important in low-turbulence environments characteristic of flight. ${ }^{9}$ The stationary crossflow vortices are are typically aligned with the potential flow producing a large fluctuating disturbance in the streamwise boundary layer profile. Crossflow vortices are also susceptible to a high frequency secondary instability that leads to rapid local breakdown, characterised by a turbulent wedge and 'saw-tooth' pattern. ${ }^{10}$ Malik et al. ${ }^{11}$ introduces a naming convention for the three different classes of secondary instability modes: (1) high-frequency ' $z$ mode' induced by the minimum of the spanwise gradient of the streamwise velocity component, located on the updraft vortex side, (2) high frequency 'y' mode, induced by the local maximum of the wall normal gradient, located on top of the vortex, and (3) 'mode III' linked to the maximum of the spanwise gradient, located close to the wall.

Direct Numerical Simulation (DNS) is playing an increasingly important role in the investigation of crossflow transition thanks to the development of highly accurate discretisation methods and the advance in high performance computing. Various DNS work has been conducted by different groups using both a temporal model and the computationally more demanding but physically more appropriate spatial model. Some recent work with spatial DNS by Wasserman \& Kloker $^{10,12}$ has successfully captured the secondary instability region using a swept flat plate with results comparing well to an independent experimental study conducted by White \& Saric. ${ }^{13}$ Kloker ${ }^{14}$ has also confirmed the potential of methods for crossflow instability suppression using smart suction methods. DNS however, is restricted to simulations at low $R e_{c}$ using simple geometries due to the heavy computational cost, for example the recent studies by Wasserman \& Kloker were run at $R e_{c}=100,000$. For this reason, it is worthwhile to investigate the application of Large Eddy Simulation (LES) to the numerical study of crossflow transition at higher $R e_{c}$. In LES the large scale eddies are resolved while the small eddies are modelled using a sub-grid scale model. While LES is intrinsically advantageous 
for modelling of flows with large scales and separation zones, advancements in sub-grid modelling and an increase in available computational resources have made it a possible route to analysis and prediction of transition.

Progress in LES transition modelling has been made in simulating natural transition in incompressible channel flow by Schlatter. ${ }^{15}$ Schlatter demonstrates the use of LES for modelling natural transition using dynamic, structure-function \& high-pass filtered sub-grid models on relatively coarse grids. The results showed that LES can successfully capture the transitional region at a fraction of the computational cost of a DNS calculation. LES has also been used previously for modelling of crossflow vortices by Huai et al. ${ }^{16}$ using a $45^{\circ}$ swept wedge. Stationary and travelling crossflow vortices were generated using steady and random amplitude suction/blowing. A dynamic Smagorinsky sub-grid model was applied (Germano et al. ${ }^{17}$ ) which lends itself to a transitional flow as the model coefficient is calculated from large-scale flow features rather than assuming a specified standard value; this allows the sub-grid scale stresses to vanish in the laminar region. The LES captured the essential features of the spatial evolution of the crossflow vortex packet and was in good agreement with DNS data.

This paper aims to study the current capability of LES to model stationary crossflow vortices on swept airfoils with validation against experimental data. Results from an experiment carried out by Chernoray et al. ${ }^{1}$ are used for this numerical study. The experiment lends itself to an LES approach as it was run at a relatively low $R_{c}(=390,000)$, but still large enough to be computationally too expensive for a DNS simulation. The experiment used a C-16 airfoil with sweep of $45^{\circ}$ and an initial baseline 3-dimensional laminar boundary layer. An artificial disturbance was then used to generate the primary instability at $0.3 x / c$ using several devices including a roughness element and continuous suction. V-probe hot wire measurements were taken at spanwise planes along the chord and the spatial evolution of the crossflow vortices were analysed. Secondary instabilities were investigated naturally and artificially (pulsed suction/blowing) with a large amount of data generated; making it ideal for numerical comparison.

The aim of the numerical study is to model the laminar boundary layer from the experiment described above and assess two LES sub-grid models. Continuous suction is then used to trigger the stationary crossflow vortex packet and compared with results from the experiment. From the results presented, conclusions are drawn as to the feasibility of using LES for modelling of crossflow transition and whether it could be used in design cycles. The paper is split into sections describing the numerical method, the computational methodology, and results of simulations of the laminar base flow and stationary crossflow vortex packet, with a final section for conclusions.

\section{Numerical Method}

\section{A. Solver}

The computational code DELTA has been used for the LES and uses a finite volume pressure-based method on a multiblock structured grid. The code has been developed at Loughborough University and has been used for both RANS and LES calculations. ${ }^{18,19}$ The code uses a high order upwind scheme (equivalent to QUICK) for spatial discretisation and a implicit backward Euler for temporal discretisation. Parallelisation is achieved by mapping the grid blocks to individual processors and updating the interface halo regions using message passing. ${ }^{20}$ A numerical transition trip can be applied to the flow at any prescribed location. The trip is applied by perturbing, at each computational time step, the flow solution in a row of computational cells near the wall. A random velocity perturbation with a Gaussian distribution and a given turbulence intensity is applied using a spatial stencil to give a weak spatial correlation to the disturbance. ${ }^{21}$

LES simulations were run in a non-dimensional scheme based upon free stream velocity $Q_{0}$ and reference length, c. The time step for LES simulations was chosen such that the maximum CFL number was less than 1 , resulting in a non-dimensional time step for the grids used in this paper of $<1.0 \times 10^{-04}$. The solver was run until 10 non-dimensional flow through times had elapsed to allow the flow to develop; subsequently statistical averages were gathered by sampling to generate a time-averaged solution

\section{B. Sub-Grid Modelling}

The success of LES for transitional flows is highly dependent on the underlying sub-grid scale (SGS) model used. The Smagorinsky model ${ }^{22}$ was used initially and assumes that the sub-grid scale viscosity is proportional to a characteristic sub-grid length $\Delta$ and to a characteristic turbulent velocity, which is taken as the 
product of $\Delta$ and the local resolved strain rate $|\bar{S}|$ magnitude, described below.

$$
\nu_{t}=\left(C_{s} \Delta\right)^{2}|\bar{S}|, \quad|\bar{S}|=\sqrt{2 \overline{S_{i j} S_{i j}}}
$$

An approximate value for $C_{s}$ is between $0.09-0.18$. The main problem with the Smagorinsky model is its behaviour near a wall. The Smagorinsky model generates a sub-grid scale viscosity wherever a velocity gradient exists, however, all turbulent fluctuations are damped near a wall, therefore $\nu_{t}$ should reduce to zero. A Van Driest ${ }^{23}$ exponential damping function can be applied along with reducing $C_{s}$ to 0.1 to sustain turbulence in channel flow; however this is an ad-hoc modification and is difficult to apply to a general case. Germano et al. ${ }^{17}$ proposed a dynamic Smagorinsky SGS model which adjusts the model coefficient to the local flow conditions e.g. reducing the model contribution near the wall in laminar and transitional regions. The dynamic model has showed promising results, however it is computationally expensive and difficult to implement for complex geometries. Results from this model also have to be clipped and adjusted for each case to provide a proper $y^{+3}$ near wall scaling for the eddy viscosity.

Nicoud \& Ducros ${ }^{24}$ subsequently proposed the Wall-Adapting Eddy Viscosity (WALE) model, that aims to recover the proper near wall scaling without a dynamic procedure and allows the eddy viscosity to tend to zero in laminar regions. The model is based on the square of the velocity gradient tensor and accounts for the effects of both the strain and rotation rate of the smallest resolved turbulent fluctuations. For the velocity gradient tensor $\bar{g}$ the model formulation is described below.

$$
\begin{gathered}
\nu_{t}=\left(C_{w} \Delta\right)^{2} \frac{\left(\mathscr{S}_{i j}^{d} \mathscr{S}_{i j}^{d}\right)^{\frac{3}{2}}}{\left(\bar{S}_{i j} \bar{S}_{i j}\right)^{\frac{5}{2}}+\left(\mathscr{S}_{i j}^{d} \mathscr{S}_{i j}^{d}\right)^{\frac{5}{4}}} \\
\mathscr{S}_{i j}^{d}=\frac{1}{2}\left(\bar{g}_{i j}^{2}+\bar{g}_{j i}^{2}\right)-\frac{1}{3} \delta_{i j} \bar{g}_{k k}^{2}, \quad \bar{g}_{i j}^{2}=\bar{g}_{i k} \bar{g}_{k j}
\end{gathered}
$$

Where $C_{w}$ is typically between $0.5-0.6$. Both the WALE and Smagorinsky model have been used in this study and the results from both are compared.

\section{Computational Model}

\section{A. Initial Solution Domain \& Boundary Conditions}

The geometry for this LES simulation is based upon the experiment of Chernoray et al. ${ }^{1}$ Figure 1 shows the experimental setup. All experimental runs were performed using a C-16 airfoil model positioned at a sweep angle of $45^{\circ}$ and with an onset velocity of $Q_{0}=8.2 \mathrm{~m} / \mathrm{s}$ giving a $R e_{c}$ of 390,000 . The chord length $\mathrm{c}$ of the configuration was $0.707 \mathrm{~m}$ with a chord measured perpendicular to the wing leading edge of $0.5 \mathrm{~m}$. The upper surface (aft of $0.4 x / \mathrm{c}$ ) of the wing is a flat surface allowing study of the flow without the presence of wall curvature effects. The wing was angled at $2^{\circ}$ positive incidence relative to the upper flat surface and a sandpaper trip was placed close to the lead-

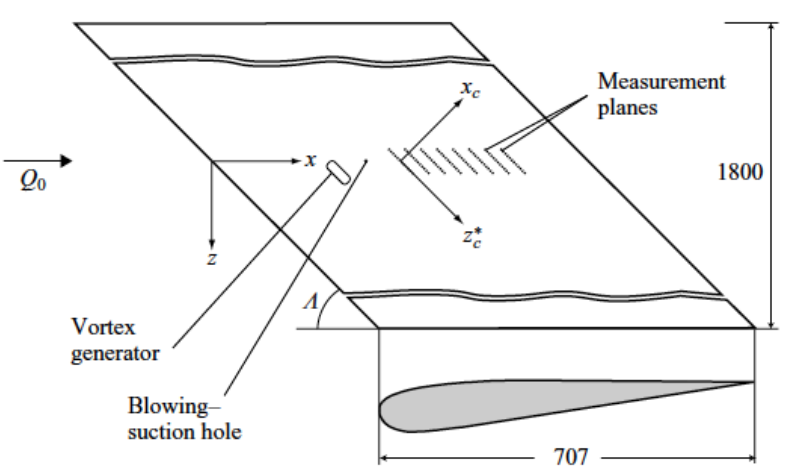

Figure 1. Chernoray ${ }^{1}$ C16 Aerofoil \& Experiment setup ing edge on the lower airfoil surface to stabilise large-scale separation and to avoid associated global unsteadiness of the flow.

The initial solution domain for the computational model is shown in Figure 2. The (x-y) plane dimensions of the domain are kept similar to that of the wind tunnel in the experiment at $5 x / c$ in the streamwise direction and $3.2 y / c$ in the transverse direction. The spanwise dimension is an important quantity for the LES; a large enough segment is required so that any turbulent eddies created including the crossflow vortices are not constrained. However a too large segment requires a much greater computational expense. To reduce the impact of the spanwise faces an infinite swept wing model was applied using a periodic or cyclic condition on the spanwise faces. This is achieved by linking the topology of each spanwise face from each block, allowing 
data to be passed from one side face back into the block on the opposite face. The spanwise wavelength of the crossflow vortices measured in the experiment was $20 \mathrm{~mm}$. A spanwise segment in the $\mathrm{Z}$ coordinate of $141 \mathrm{~mm}(0.2 \mathrm{c})$, with $45^{\circ}$ sweep allows for greater than 10 wavelengths of the crossflow vortices. The impact of the spanwise dimension was investigated and increasing the span showed no further change to the results presented. The angle of attack was adjusted so that no laminar separation occurred on the upper surface, as was the process in the experiment. The final angle of the upper surface was $0^{\circ}$.

Simulations were initialised with an inlet velocity of $Q_{0}=8.2 \mathrm{~m} / \mathrm{s}$ and a numerical trip with turbulent intensity $0.1 \%$ of $Q_{0}$ was applied near the inlet to provide a low freestream turbulent environment. A numerical trip was also applied near the leading edge on the lower surface to trip the boundary layer from laminar to turbulent to reduce and control the unsteadiness of any generated separation.

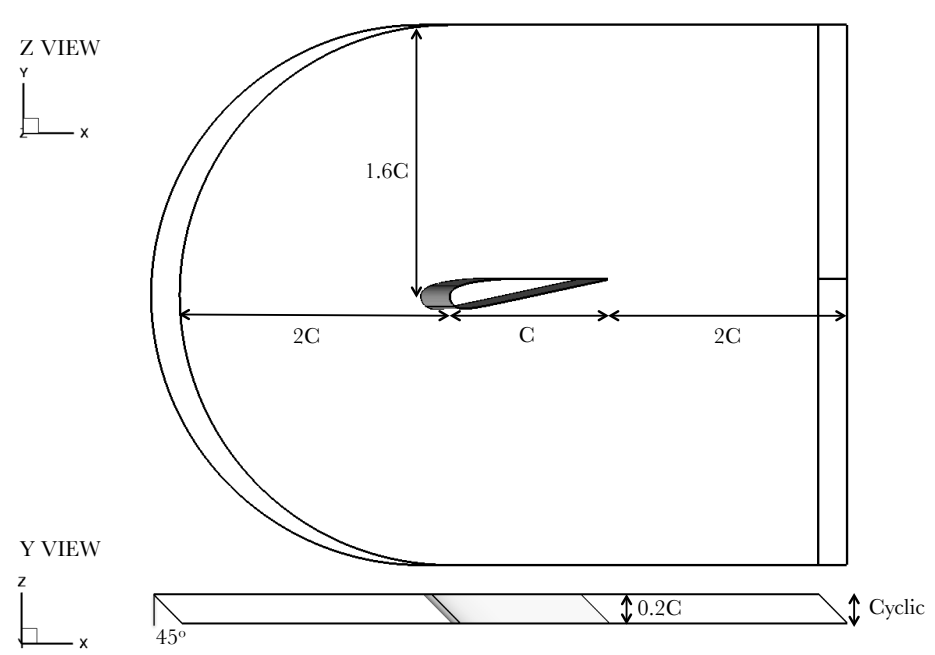

Figure 2. Initial Solution Domain

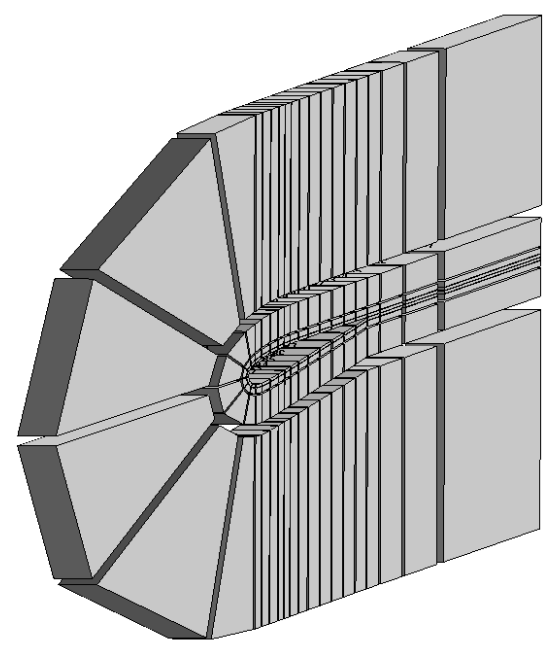

Figure 3. Initial Block Structure

\section{B. Initial Grid \& Streamline Extraction}

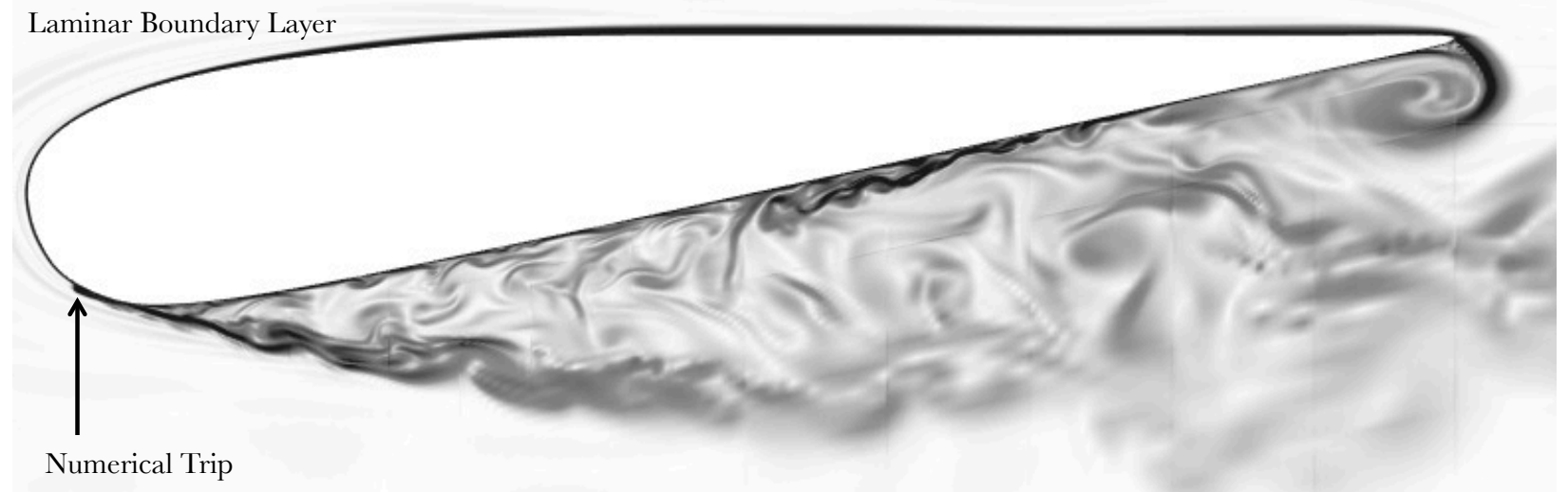

Figure 4. Instantaneous Vorticity Magnitude at 50\% Span, Scale: White - 0 Black - 150

An initial C grid with 152 blocks was generated within the solution domain shown in Figure 2; the block structure is shown in Figure 3. The grid consists of 700 nodes on each of the upper and lower surface of the airfoil with 160 nodes in the wall normal direction. 140 nodes extend towards the outlet and the span 
contains 320 nodes. For the $x$-coordinate the leading edge $\Delta x+=30$ which is reduced to 20 at $x / c=0.3$ where the suction hole was placed; the mesh is expanded at rate 1.05 to $\Delta x+=60$ towards the trailing edge. $\Delta y+=1.5$ at the wall with a slow wall normal expansion rate of 1.06 , the $z$-spacing was kept uniform with a $\Delta z+=22$. The resulting mesh consists of 75 million nodes. Piomelli \& Balaras ${ }^{25}$ advise on the necessary grid requirements for resolving the wall using LES and the initial grid meets the criteria described $(\Delta x+<100$ and $\Delta z+<20)$.

The initial grid was run to examine preliminary flow field predictions and to assess whether the full solution domain could be simplified. Figure 4 shows an instantaneous plot of vorticity magnitude for the initial grid. The airfoil upper surface shows clearly a laminar boundary layer extending across the full length of the chord. The position of the numerical trip is shown on the lower surface and clear breakdown to turbulence can be seen. The lower surface shows separation at $0.8 x / c$ with large scale unsteadiness. No results from the lower surface were presented in the experiment and as mentioned above a sandpaper trip was applied near the leading edge to stabilise lower surface separation and to reduce any impact on the upper surface. For this case, the lower surface is not essential for analysis of the crossflow vortices on the upper surface. By removing the need to simulate the lower surface flow the saving in computational resources can be used to refine the simulation, in order to better resolve the crossflow vortices on the upper surface. The experiment also aimed at reducing the impact of the lower surface by tripping the boundary layer, therefore by removing the lower surface flow without impacting on the accuracy the same result can be achieved.

The method used to remove the lower surface was to export from the steady, time averaged mean solution the stagnation streamline upstream of the leading edge and the streamline aft of the trailing edge. These were averaged across the span and converted (together with the geometry of the upper airfoil surface) into a plane which defined the inner boundary of a new solution domain. The upper and downstream boundaries remained the same as the initial (full C-16) solution domain. Figure 5 shows the extracted streamlines from the initial grid and the conversion into the final streamline solution domain. Note, it can reasonably be expected that the onset flow stagnation streamline will not fluctuate in time. Downstream of the trailing edge the flow will almost certainly be unsteady, and this effect is absent in the small solution domain since the mean streamline is used. However this is only likely to affect the simulation at the rear half of the upper surface and the behaviour of the crossflow vortex packet should not be unduly influenced.

The upper half of the initial grid was placed upon the streamline solution domain, reducing the overall node count by a half. The grid on the streamline geometry consists of 76 blocks with 38 million nodes. The streamline geometry was then run with a slip wall boundary condition placed on the streamline surfaces. To examine whether the streamline geometry results were equal to those from the full geometry, results of pressure coefficient, laminar boundary layer profiles and wall shear stress are compared, shown in Figure 6. The comparison shows that upper surface velocity profiles are nearly identical up until $0.5 x / c$. Aft of $0.5 x / c$ the streamline model laminar boundary layer velocity profiles take a slightly different shape to that of the initial geometry presumably for the reason mentioned above. The pressure gradient aft of $0.5 x / c$ is shallower for the streamline case as is the skin friction coefficient. It was decided that the discrepancy between the initial geometry and the streamline geometry is small enough to use the streamline grid for further analysis, especially as the results are nearly identical in the region of interest $0.3-0.5 x / c$. The benefit of simplification of the geometry and overall reduction in computational cost outweigh the small discrepancies in accuracy. 


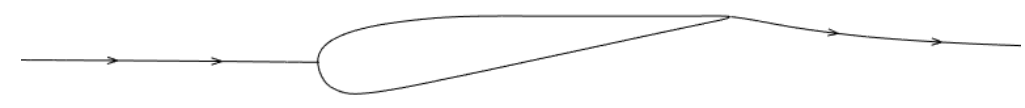

(a) Span averaged extracted Initial Grid Streamlines

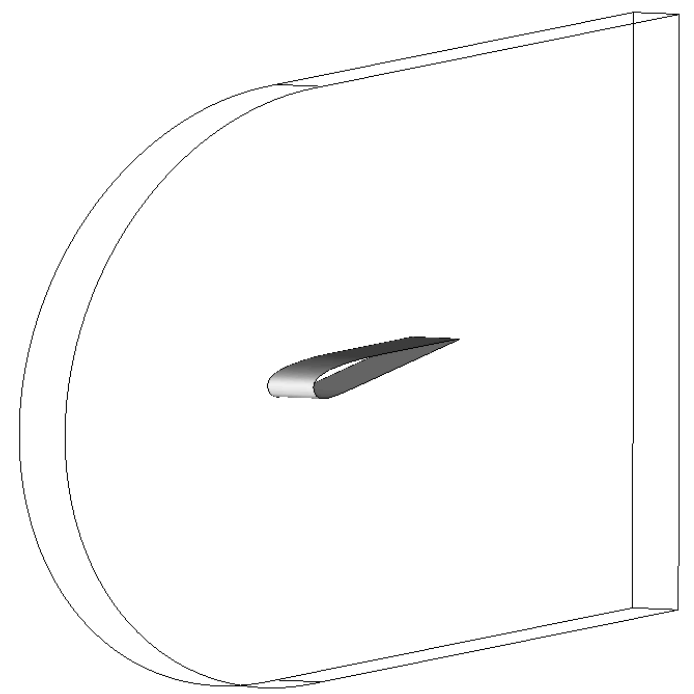

(b) Initial Solution Domain

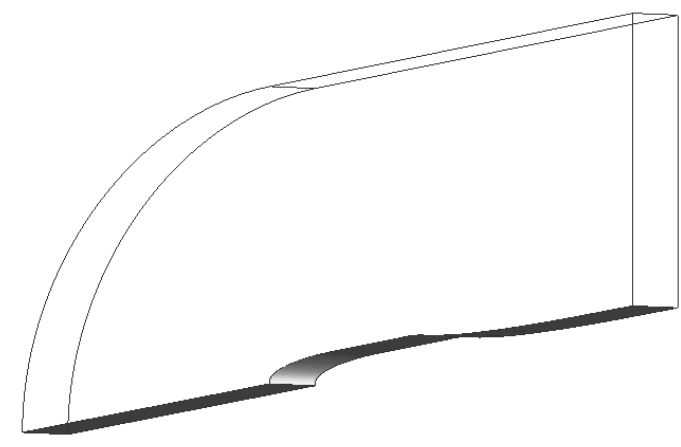

(c) Streamline Solution Domain

Figure 5. Streamline Extraction

\section{Fine Grid}

A refined grid was generated to better resolve the region aft of the continuous suction hole at $0.3 x / c$. The streamwise and spanwise spacing were greatly reduced to increase the ratio of resolved/modelled scales in the LES. The fine grid has 300 blocks and consists of 1033 nodes on the upper airfoil surface with 190 nodes in the wall normal direction. 175 nodes extend towards the outlet and the span contains 700 nodes. In the $x$-coordinate the leading edge $\Delta x+=20$ and reduces to $\Delta x+=6$ at $x / c=0.3$ where the suction hole is placed, the mesh is then gradually expanded to $\Delta x+=40$ towards the trailing edge. The near wall $\Delta y+=1.0$ with a slow wall normal expansion rate of 1.06 , the $z$-spacing was kept uniform with a $\Delta z+=12$. The resulting mesh consists of 161 million nodes. Both the initial and fine grid were used for modelling the crossflow vortex case and both grids are summarised in Table 1.

Table 1. Grid Diagnostics

\begin{tabular}{lrr}
\hline \hline & Initial Grid & Fine Grid \\
\hline Block Count & 76 & 300 \\
Airfoil Nodes & 700 & 1033 \\
Wall Normal Nodes & 140 & 190 \\
Spanwise Nodes & 320 & 700 \\
Leading Edge, $\Delta x+$ & 30 & 20 \\
$0.3 x / c, \Delta x+$ & 20 & 6 \\
Trailing Edge, $\Delta x+$ & 60 & 40 \\
$\Delta y+$ & 1.5 & 1.0 \\
$\Delta z+$ & 22 & 12 \\
Total Mesh Size & $38 \mathrm{mil}$ & $161 \mathrm{mil}$ \\
\hline
\end{tabular}




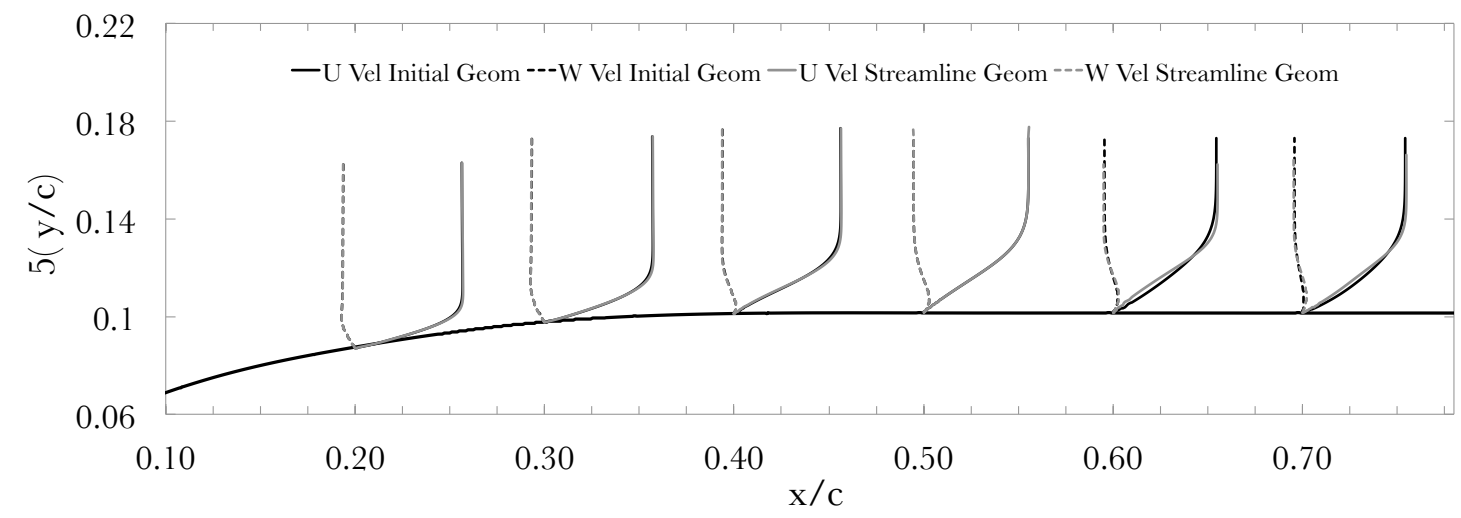

(a) Velocity profiles for Initial \& Streamline solution domain along chord (Note: $y / c$ is 5 times magnified for clarity)

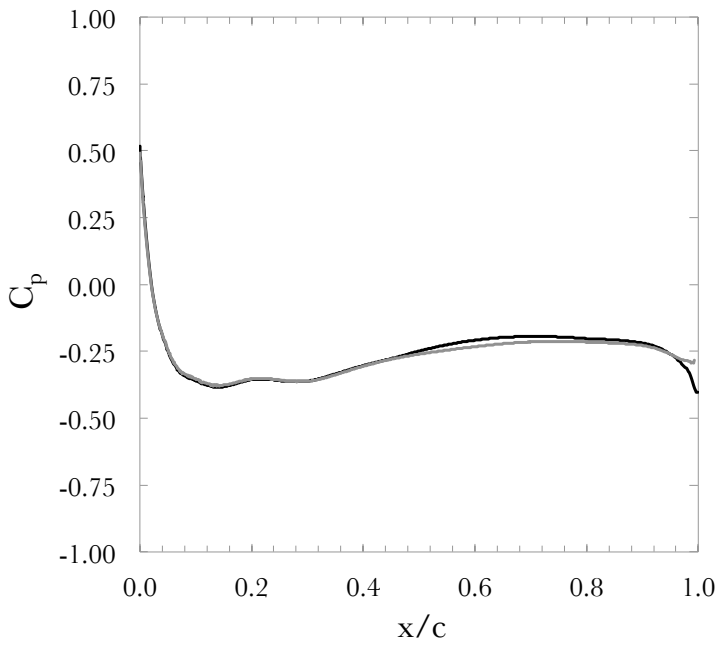

(b) Pressure Coefficent

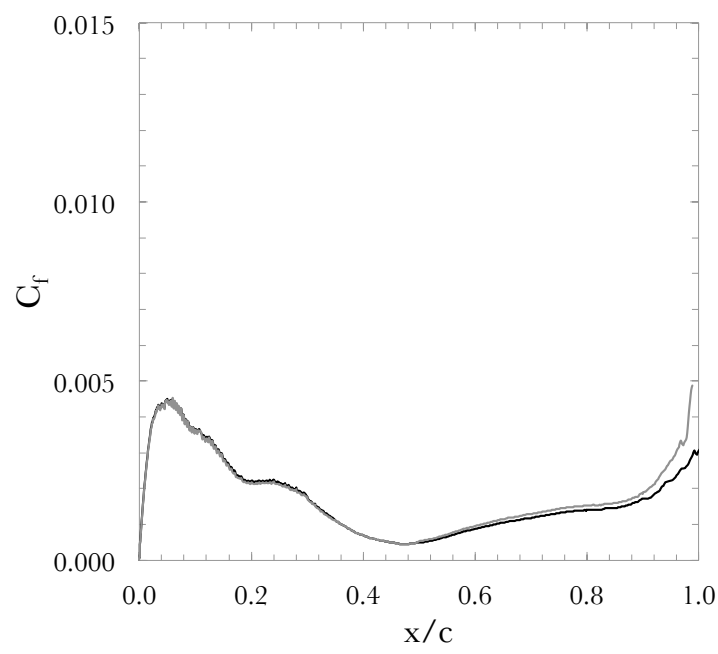

(c) Skin Friction Coefficent

Figure 6. Comparison of Initial \& Streamline Geometry, Grey - Streamline, Black - Initial 


\section{Results}

\section{A. Laminar Base Flow}

With no artificial disturbance excited a typical Falker-Skan-Cooke (FSC) 3-dimensional laminar boundary layer flow was formed on the upper surface with an initial accelerated region followed by an area of deceleration. Chernoray et al. ${ }^{1}$ compares measured velocity profiles at $0.3 x / c$ and results approximate to a FSC similarity solution giving $R e_{\theta}=175$ at $0.3 x / c$. Aft of 0.5 chord a self- similar FSC boundary layer is formed. Results from the LES simulations for both initial and fine grid for the laminar base flow are illustrated in Figure 7.

Figure 7 (a) shows velocity profiles taken at $0.3 x / c$ from the experiment and compared to mean sampled LES results. The figure shows LES profiles using the Smagorinsky model (initial grid) and WALE model (initial \& fine grid). The figure shows that the Smagorinsky model over predicts the boundary layer thickness by $30 \%$ while the WALE model produces a profile closer to the experimental points. Figure 7(d) shows the ratio of eddy viscosity to molecular viscosity against distance to the wall at $0.3 x / c$. The figure shows that the Smagorinsky model produces a large amount of eddy viscosity near the wall, reducing the effective local Reynolds number and resulting in an increased boundary layer thickness. The WALE model however produces a near zero eddy viscosity near to the wall, essentially becoming a real laminar flow. Figure 7(e) shows boundary layer profiles at different locations along the chord and shows the difference between the laminar boundary layer profiles for the initial grid. The WALE model consistently predicts a lower boundary layer thickness along the whole airfoil chord.

Figure 7(b) compares experimental $\mathrm{U} \& \mathrm{~W}$ Velocities at a line $1 \mathrm{~cm}$ above the airfoil with the WALE fine grid results (Note: results were identical for both the initial \& fine grid with both Smagorinsky \& WALE models). The results show a strong correlation to the the experimental data although the LES has a steeper velocity gradient at the leading edge. Figure $7(\mathrm{c})$ shows a comparison of the momentum thickness $(\theta)$ from the experimental results with the fine LES solution. The results show a stronger gradient of momentum thickness along the chord compared to the experiment however results from the LES are within $10 \%$ from $0.3-0.6 x / c$.

It was decided that the LES results of the laminar base flow were sufficiently accurate compared to the laminar base flow as described in the experimental data of Chernoray et al. ${ }^{1}$ LES results were then obtained focusing on stationary crossflow vortices within the laminar boundary layer, the results of which are described in the next section.

\section{B. Stationary Crossflow Vortex Packet}

A packet of stationary vortex modes can be generated by strong localised continuous suction; this was demonstrated in the experimental test case. Cheronray et al. ${ }^{1}$ generated the primary instability of the crossflow vortex modes using a $1 \mathrm{~mm}$ diameter hole with a high suction rate (order of magnitude $Q_{0}$ ) located at $0.3 x / c$. The LES results aim to replicate the experimental procedure and to adjust the suction rate until the crossflow vortices are of similar wavelength to that of the experiment. For the LES a $1 \mathrm{~mm}$ diameter hole was applied with a suction rate of $3 Q_{0}$; results are presented for the initial and fine grid using the WALE model. The results in this section are described in a local axis $\left(x^{*}, y^{*}, z^{*}\right)$ where the origin $(0,0,0)$ is placed at the point of suction and $\left(x^{*}, y^{*}, z^{*}\right)$ remain aligned with the global $(x, y, z)$. Figure 9 shows the position of the local axis and the domain results are presented in this section.

The suction hole acts on the flow much like a roughness element, causing a strong $\mathrm{W}$ velocity component leading to vortices of opposite rotation either side of the suction hole. This is shown in Figure 8 with a contour plot of $\mathrm{W}$ velocity on a z-plane at $0 x^{*} / c$ (above suction hole) using the fine grid. The figure shows the development of the primary instability of the crossflow vortices with strong positive and negative $\mathrm{W}$ velocity either side of a suction hole at $z^{*} / c=0$. The strong velocity and primary instability can be shown on the figure to be deep within the boundary layer, below $6 \theta$.

Visualisation of the vortices is demonstrated by iso-surfaces of $\lambda_{2}$ in Figures $10 \& 11$. The $\lambda_{2}$-definition is used to define vortical structures and was developed and described by Jeong \& Hussain. ${ }^{26} \lambda_{2}$ is a real eigenvalue of the tensor $\mathrm{S}^{2}+\Omega^{2}$ where $\mathrm{S}$ and $\Omega$ are the symmetric and antisymmetric parts respectively of the velocity gradient tensor $\bar{g}$. As $\mathrm{S}^{2}+\Omega^{2}$ is real and symmetric, it has only real eigenvalues. With $\lambda_{1}, \lambda_{2}$ and $\lambda_{3}$ as the eigenvalues such that $\lambda_{1} \geq \lambda_{2} \geq \lambda_{3}$, a vortex core is defined as a region of having a negative value of $\lambda_{2}$. This definition captures the pressure minimum in a plane perpendicular to the vortex axis. 


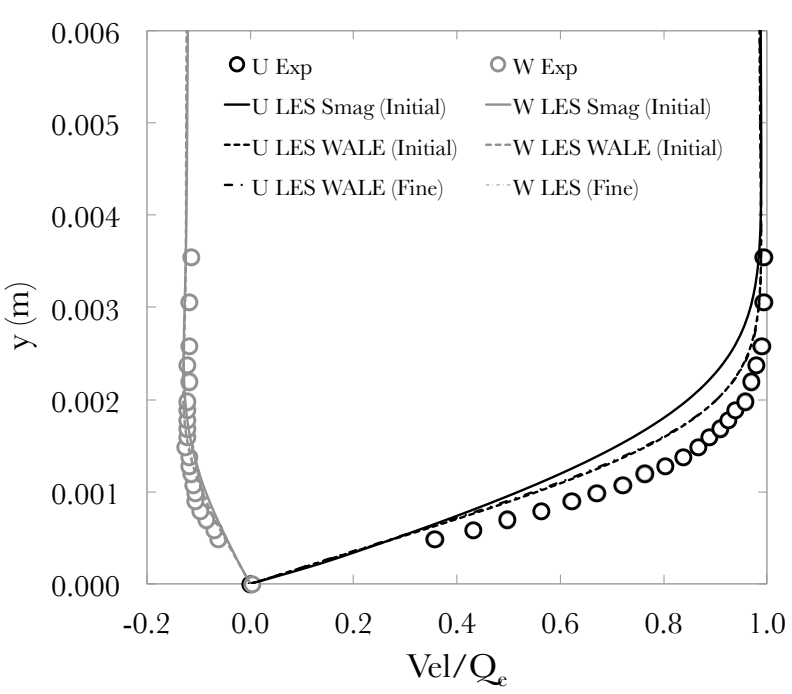

(a) Laminar boundary layer profile at $0.3 x / c$

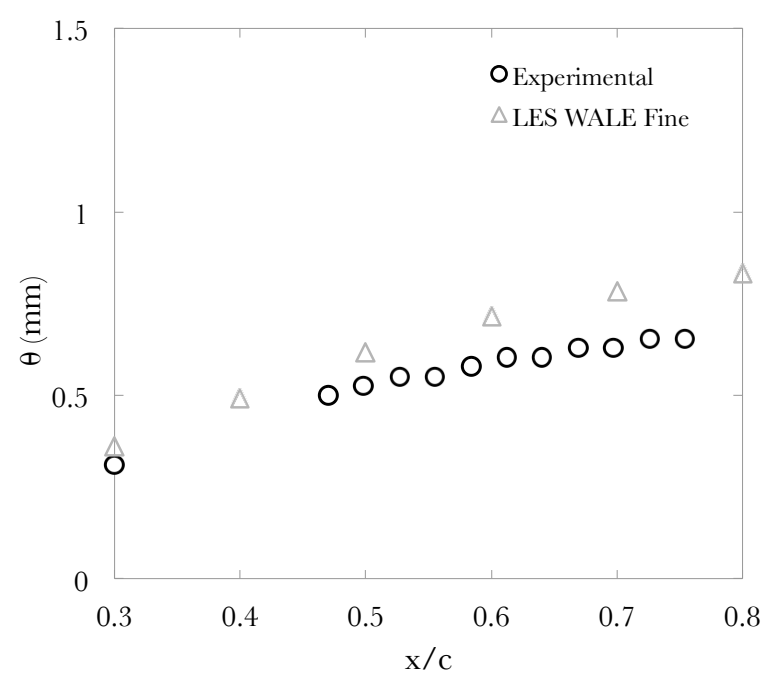

(c) Momentum Thickness $\theta$ for experiment \& LES

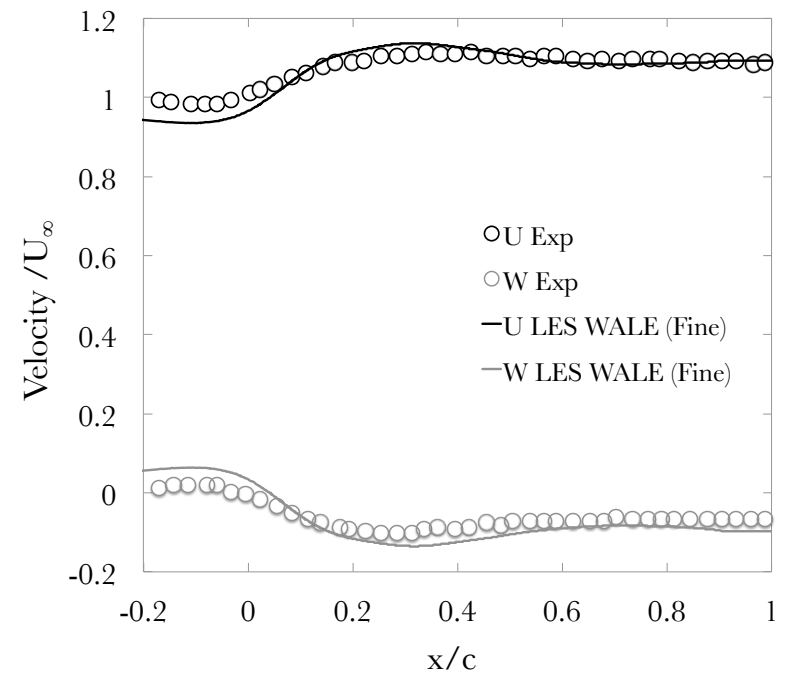

(b) U \& W Velocity at $1 \mathrm{~cm}$ above airfoil

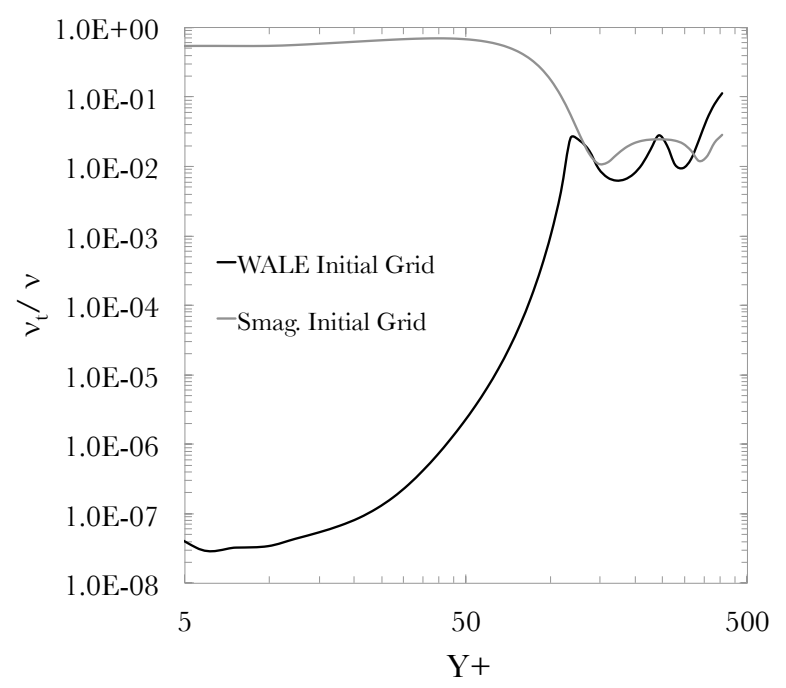

(d) Ratio of eddy-viscosity $\nu_{t}$ to molecular viscosity $\nu$ against wall distance $(y+)$ at $0.3 x / c$

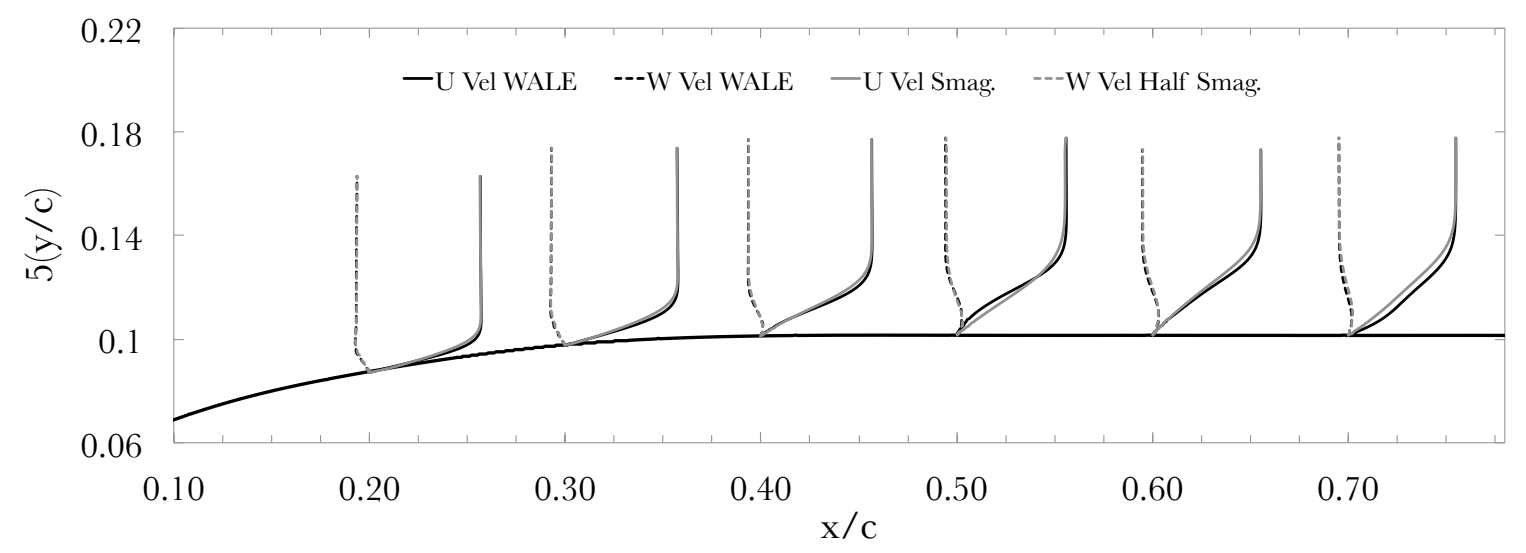

(e) Velocity profiles for Initial Grid WALE \& Smagorinsky model across chord (Note: $y / c$ is 5 times value)

Figure 7. Analysis of laminar base flow \& comparison to experimental data 


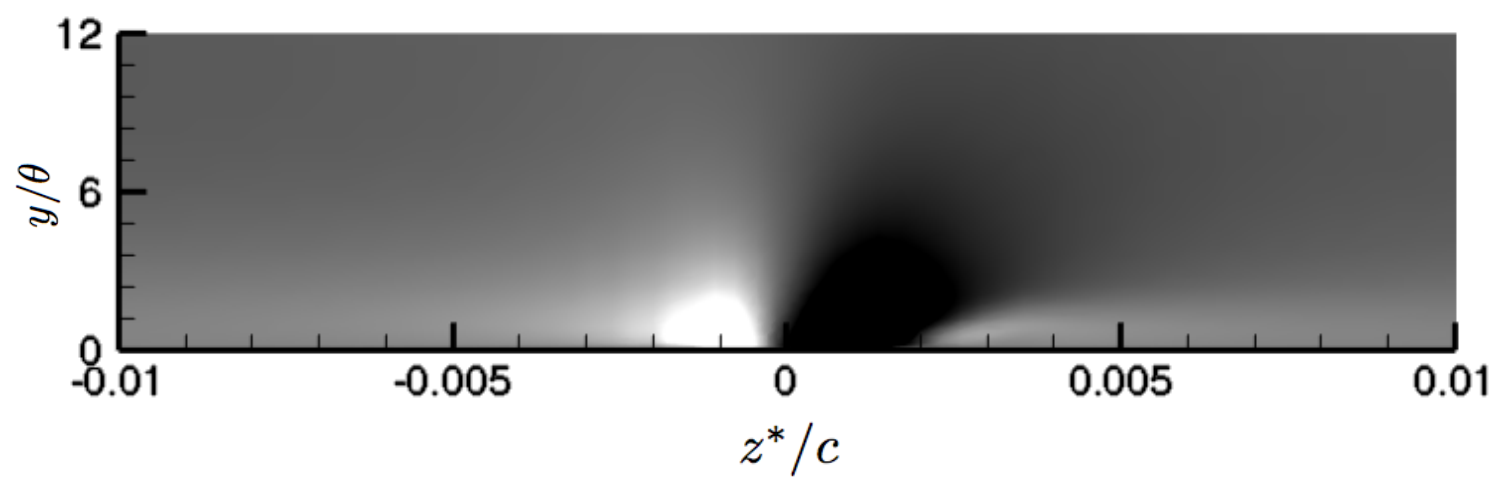

Figure 8. Contour plot of $\mathbf{W}$ Velocity on at station $x^{*} / c=0$ (Fine Grid) $\theta=\mathbf{0 . 3 1 m m}$ at this station Scale Black $=-0.5 / Q_{0}$ White $=0.5 / Q_{0}$

Figure 10 shows $\lambda_{2}$ iso-surfaces for both initial and fine grids, Figure 11 shows the same information plotted on the $y=0$ view. Between $0-0.1 x * / c$ the packet of crossflow vortices can be shown to develop either side of the suction hole with varying amplitudes, for both grids. The vortex packet on the left hand side for both initial and fine grids amplies, developing a secondary instability and eventually breaks down to turbulence. The crossflow mode on the right of the suction hole can also be visualised but is of lower amplitude to the left hand side. The vortices on the right can be shown to eventually interact with the turbulent region on the left hand side in the Figure.

The initial grid shows breakdown of the left crossflow vortex packet at $0.2 x^{*} / c$ with an 'explosive' secondary instability. Aft of $0.2 x^{*} / c$ clear hairpin structures can be shown to form within a large turbulent wedge formation; the latter is typical of crossflow induced transition. The hairpin structures have a strong negative $\mathrm{W}$ velocity component and extend as far back as $0.4 x^{*} / c$ with clear vortical streaks forming closer to the surface of the airfoil. For the fine grid, breakdown of the crossflow vortex can be identified closer to $0.3 x^{*} / c$. The fine grid also shows a turbulent wedge formation with vortical streaks of finer scales but no evidence of hairpin vortices, it is clear from the figure that the turbulent wedge region has a much larger span wise growth rate for the initial grid than on the fine grid.

Chernoray et al. ${ }^{1}$ showed natural secondary instabilities occurring at $0.28 x^{*} / c$ which is similar to that predicted in the fine grid simulation. The figure shows that for both initial \& fine grids the vortical disturbances on the left with anti-clockwise rotation are more favourable to secondary instability and breakdown to turbulence. The vortical structures on the right are shown to be much more favourable with clockwise rotation against the $\mathrm{W}$ component of the mean flow.

Figure 12 is a contour plot of instantaneous $\mathrm{Y}$ vorticity on the surface of the airfoil from $x^{*} / c 0$ - 0.6 for the initial \& fine grid. The plot shows clearly the path taken by the crossflow vortex packets either side of the suction hole. For the initial grid the crossflow vortex on the left hand side of the suction hole undergoes a secondary instability at $0.21 x^{*} / c$; downstream of this location the $\mathrm{Y}$ vorticity from the left and right crossflow modes overlap causing an early explosive break down to turbulence. Due to this the formation of large scale hairpin vortices develops within a much wider turbulent region. The coarser grid spacing in the initial grid cannot resolve the growth of the crossflow vortices correctly.

The fine grid shows secondary instabilities occurring at $0.3 x^{*} / c$ in Figure 12. The vortex packet at the right of the suction hole remains independent of the packet on the left and is stable until the turbulent wedge region of the left crossflow mode at $0.36 x^{*} / c$. It is clear that the coarse LES grid is not capable of modelling the development of the crossflow packet adequately.

The experimental data from Chernoray et al. ${ }^{1}$ included a plot of the mean streamwise velocity disturbance at $x^{*} / c=0.28$ aft of the continuous suction hole. This is calculated by removing the mean $\mathrm{U}$ velocity of the laminar base flow from the mean $U$ velocity from the suction-disturbed flow. Figure 13 shows the experimental contour plot of U' at $0.28 x^{*} / c$ and for the fine LES grid at stations $0.05,0.10,0.22 \& 0.28$ $x^{*} / c$. On the right hand side of the figure, contour plots of $u_{r m s}$ for the suction-disturbed flow are shown. The plots show clearly the growth of the crossflow vortex packet disturbance structure within the boundary layer. The fine LES results show a good correlation to the experimental plot although the experiment predicts 
a disturbance deeper in the boundary layer at $0.28 x^{*} / c$. This may however be misleading as the contour levels were not identified in the experiment. The shape of the disturbance can be explained by looking at the contour plots on the right of Figure 13. The plots of $u_{r m s}$ at $0.05 x^{*} / c$ show a packet of 3 clear crossflow modes and turbulent fluctuations. Along the chord the stationary crossflow modes grow, amplify and interact. At $0.1 \& 0.22 x^{*} / c$ the packet of crossflow modes has saturated into two clear vortical structures which have amplified greatly. At $0.28 x^{*} / c$ secondary instabilities occur and breakdown to turbulence is observed soon after. The saturation of crossflow modes within a packet has also been identified by Chernoray et al. ${ }^{1}$ and Wassermann \& Kloker $^{10,12}$ demonstrated that packets of crossflow modes are more favourable to secondary instability than a single mode, both of which can be seen in the results shown.

Figure 14 shows instantaneous plots of the spanwise $\frac{d U}{d z}$ and wall normal gradients $\frac{d U}{d z}$ with superimposed $u_{r m s}$ contours at stations $x^{*} / c 0.22,0.28,0.3,0.32$. From the laminar base flow shown in Figure $7(\mathrm{e})$ the spanwise velocity component becomes inflectional at $0.5 x / c\left(0.2 x^{*} / c\right)$ which prompts the onset of the secondary instability. Figure 14 shows the linear development of the secondary instability aft of inflection with increasing amplitudes of the ' $\mathrm{z}$ ' and ' $\mathrm{y}$ ' modes. At $0.3 x^{*} / c$ the ' $\mathrm{z}$ ' mode begins to breakdown causing a non-linear breakdown of the velocity gradient components.

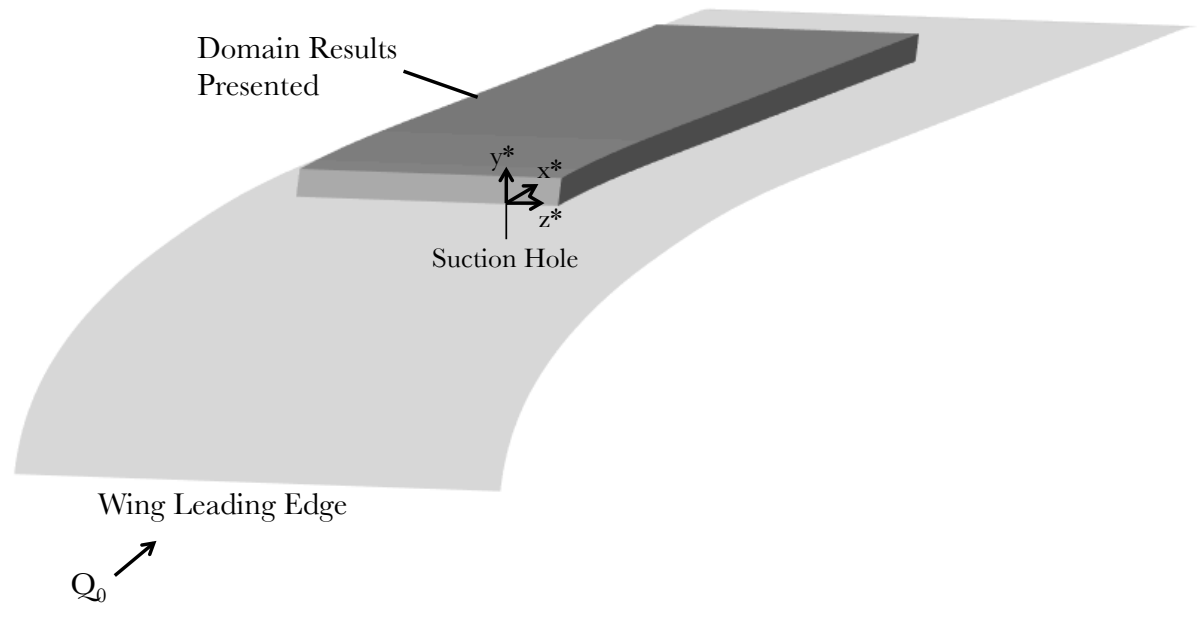

Figure 9. Post Processing Domain 


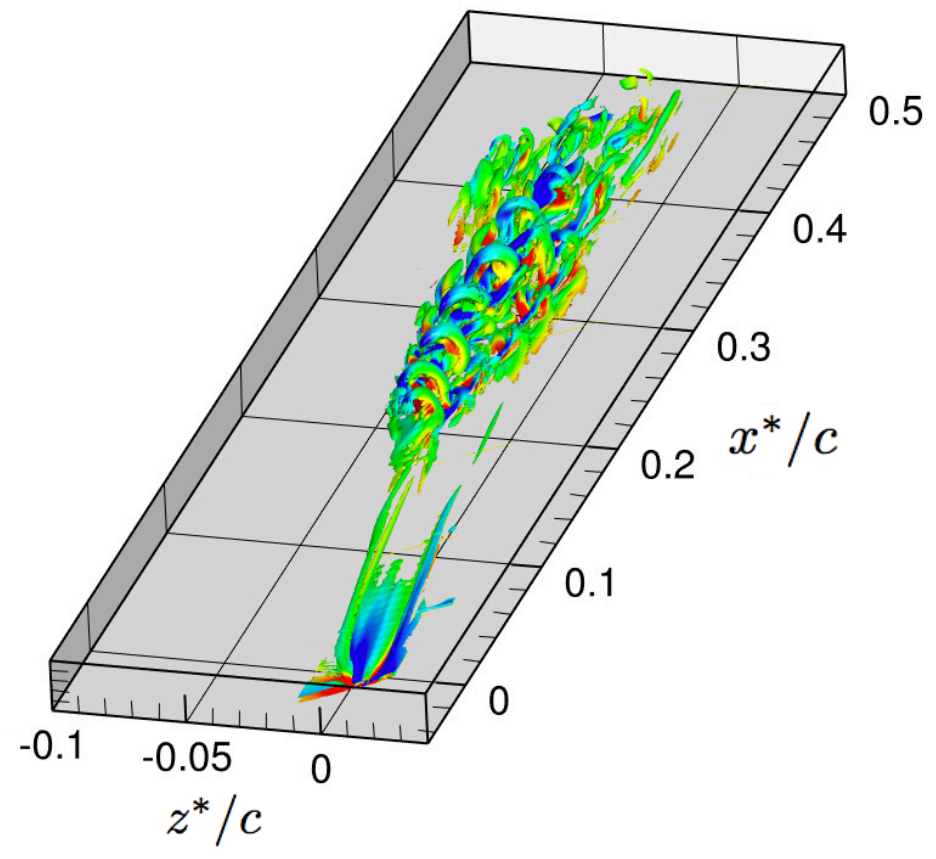

(a) Initial Grid

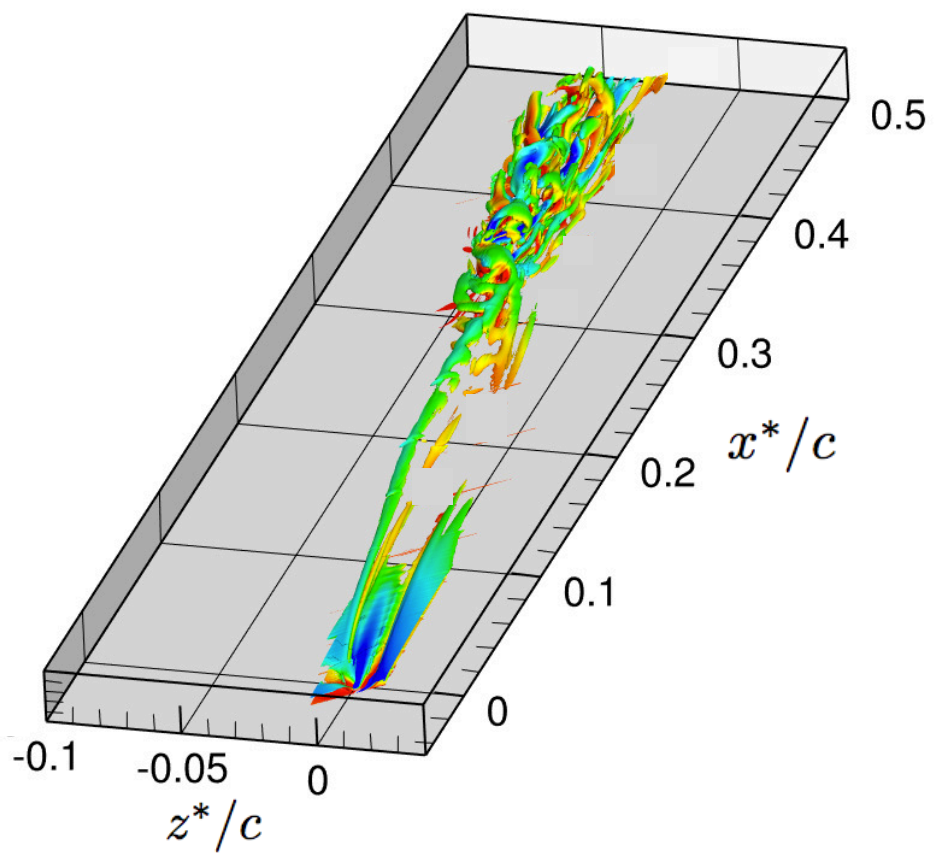

(b) Fine Grid

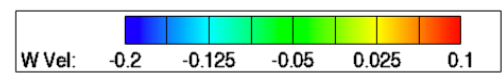

Figure 10. Visualization of vortical structures within boundary layer aft of suction hole by instantaneous $\lambda_{2}$-isosurfaces, coloured by $\mathbf{W}$ velocity $\left(\lambda_{2}=-150\right)$ (Isometric view) 


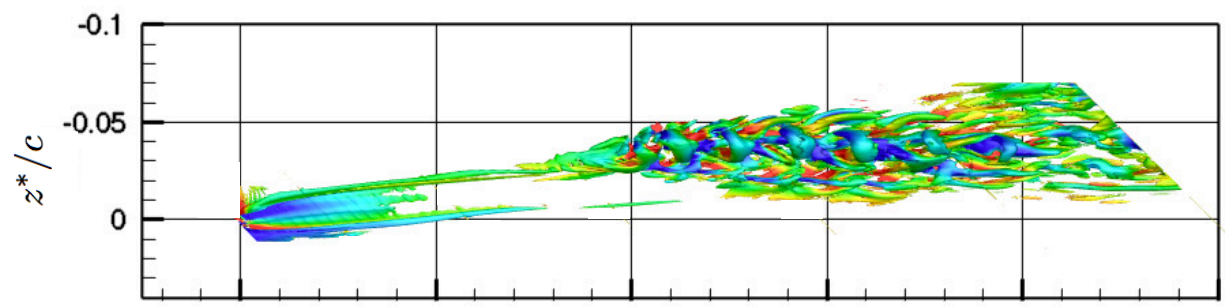

(a) Initial Grid

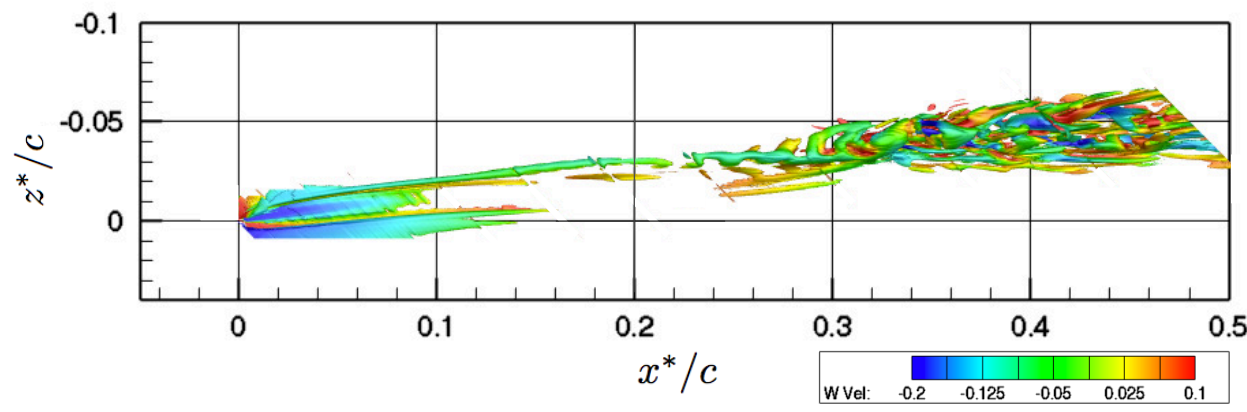

(b) Fine Grid

Figure 11. Visualization of vortical structures within boundary layer aft of suction hole by instantaneous $\lambda_{2}$-isosurfaces, coloured by $\mathbf{W}$ velocity $\left(\lambda_{2}=-150\right)(y=0$ axis view $)$

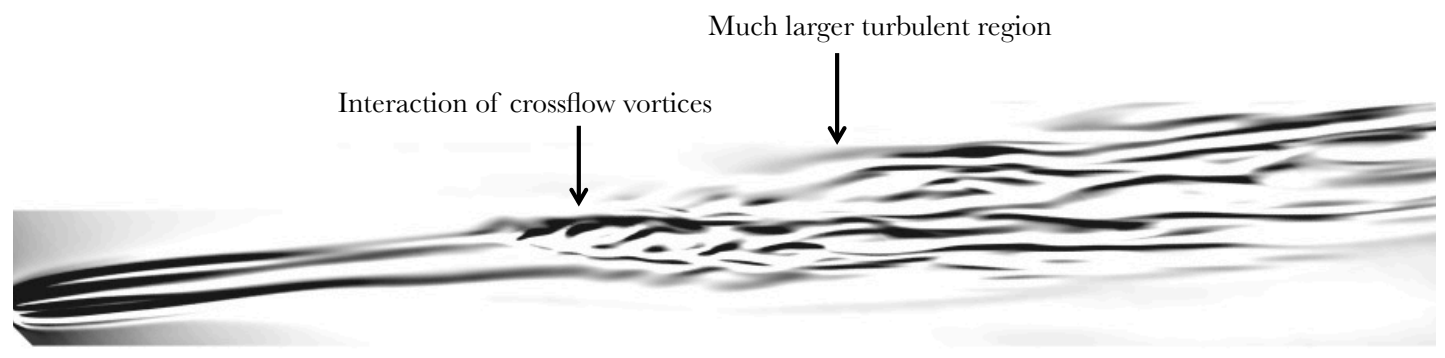

(a) Initial Grid

Artificial Primary Instability

Explosive Secondary Instability

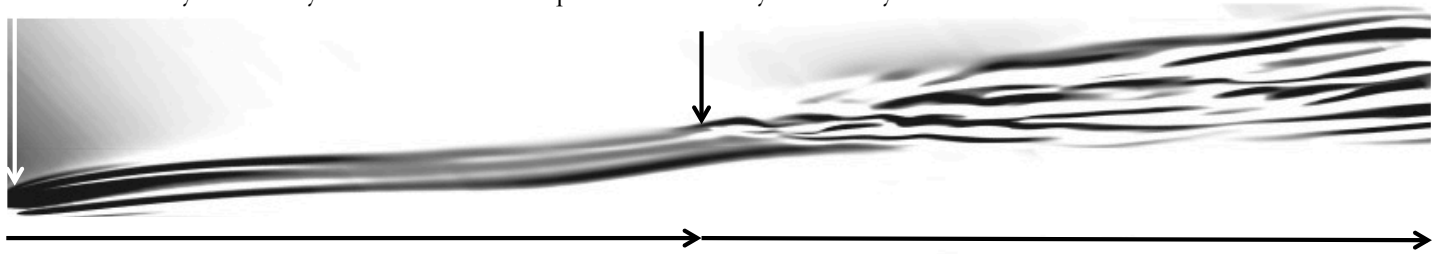

Growth \& Development of Crossflow Vortices

Turbulent Streaks in Wedge Formation

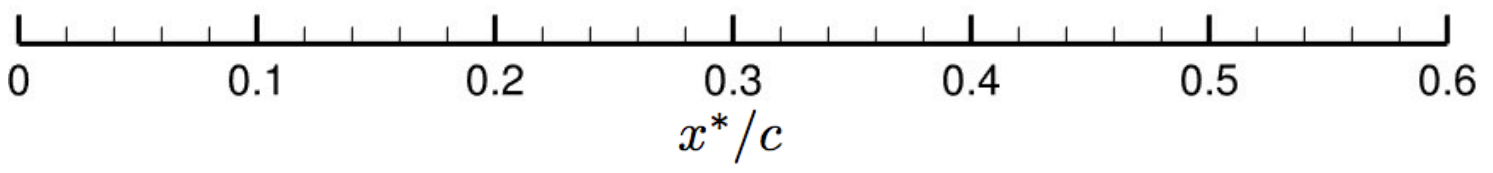

(b) Fine Grid

Figure 12. Instantaneous Y Vorticity plot on surface of airfoil for both initial \& fine grid, Scale - White - 0 Black - 25 


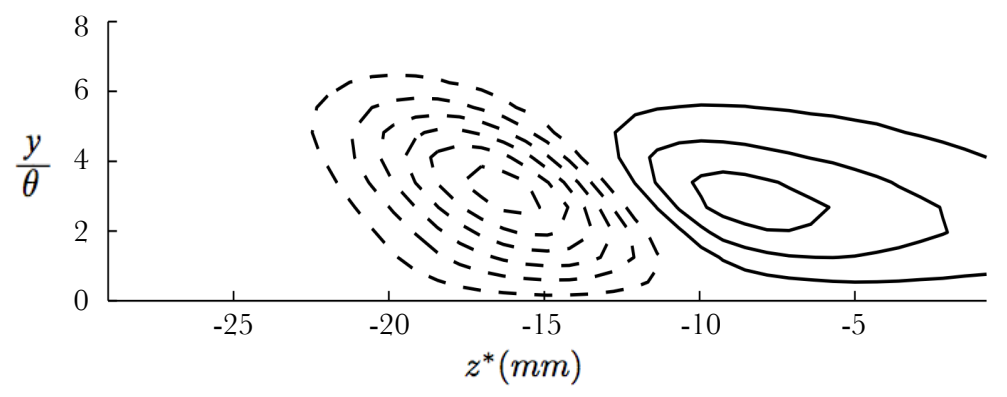

(a) Chernoray et al. ${ }^{1} 0.28 x^{*} / c$
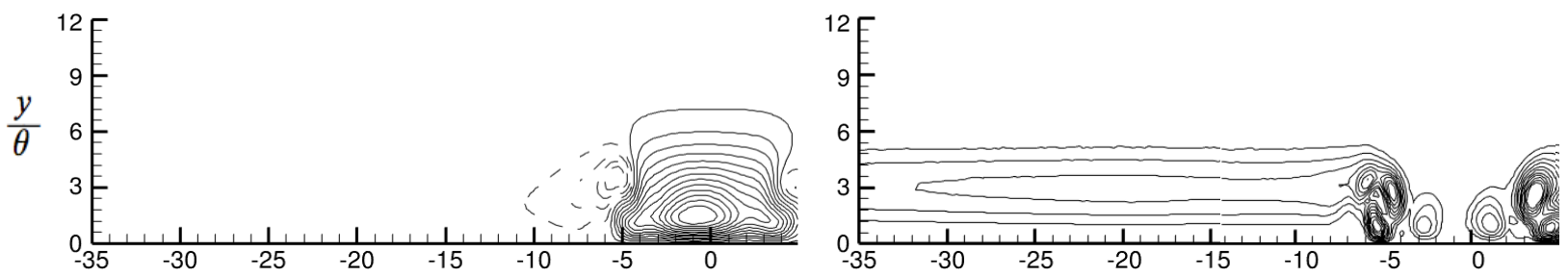

(b) Left: U' Right: $u_{r m s}$ at $0.05 x^{*} / c$
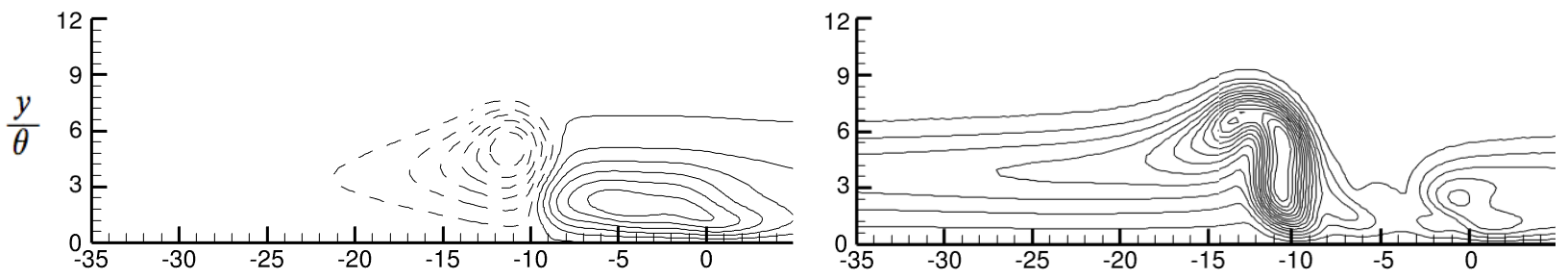

(c) Left: U' Right: $u_{r m s}$ at $0.10 x^{*} / c$
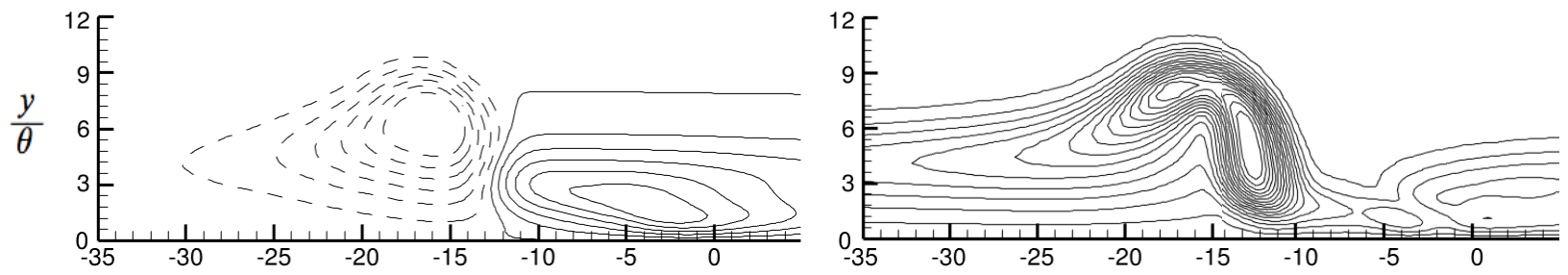

(d) Left: U' Right: $u_{r m s}$ at $0.22 x^{*} / c$
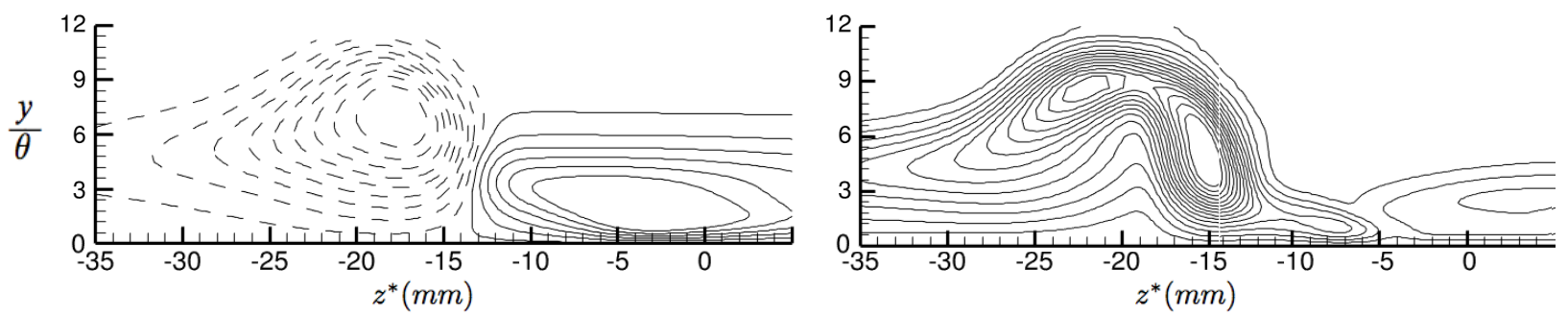

(e) Left: U' Right: $u_{r m s}$ at $0.28 x^{*} / c$

Figure 13. Mean streamwise velocity disturbance $U^{\prime}$ and $u_{r m s}$ at various $x^{*} / c$ stations (Dashed lines represent negative) $\left(u_{r m s}\right.$ scale: Contour lines from $\left.0-0.3 / Q_{0}\right)$ 

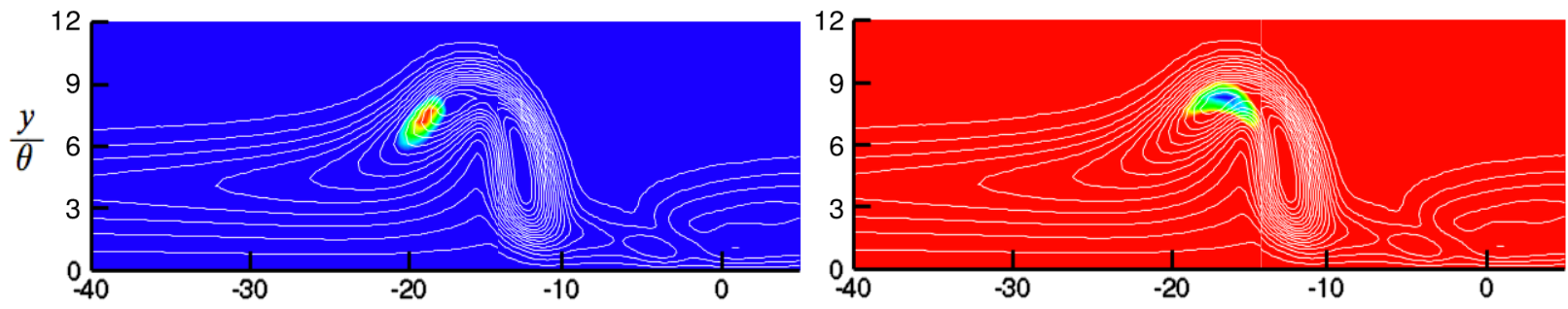

(a) $0.22 x^{*} / c$
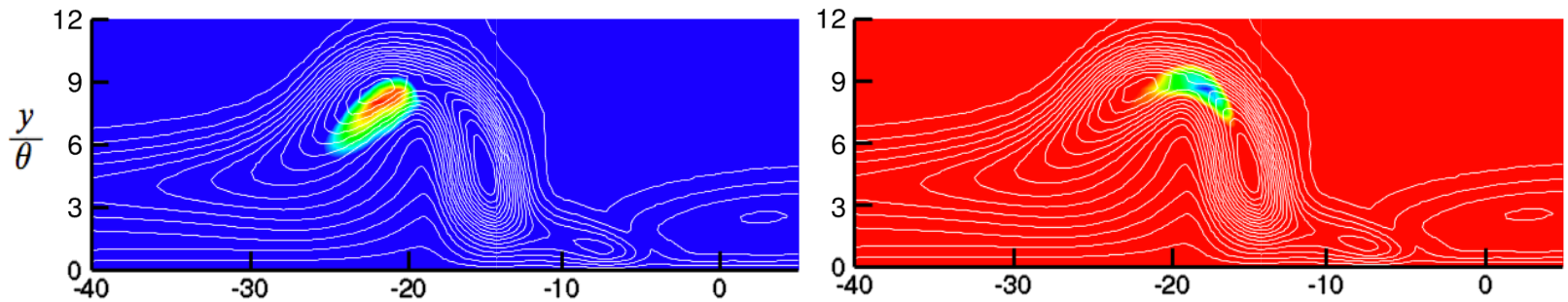

(b) $0.28 x^{*} / c$
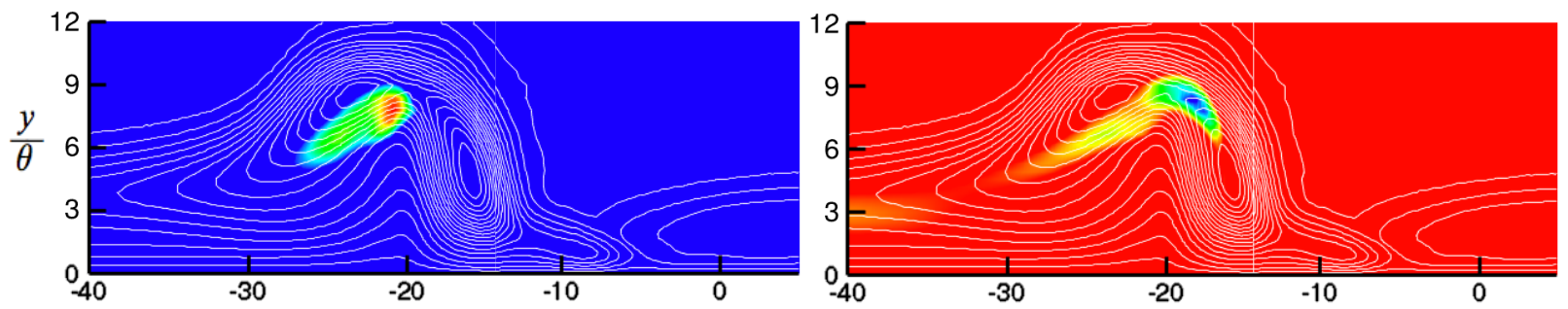

(c) $0.30 x^{*} / c$
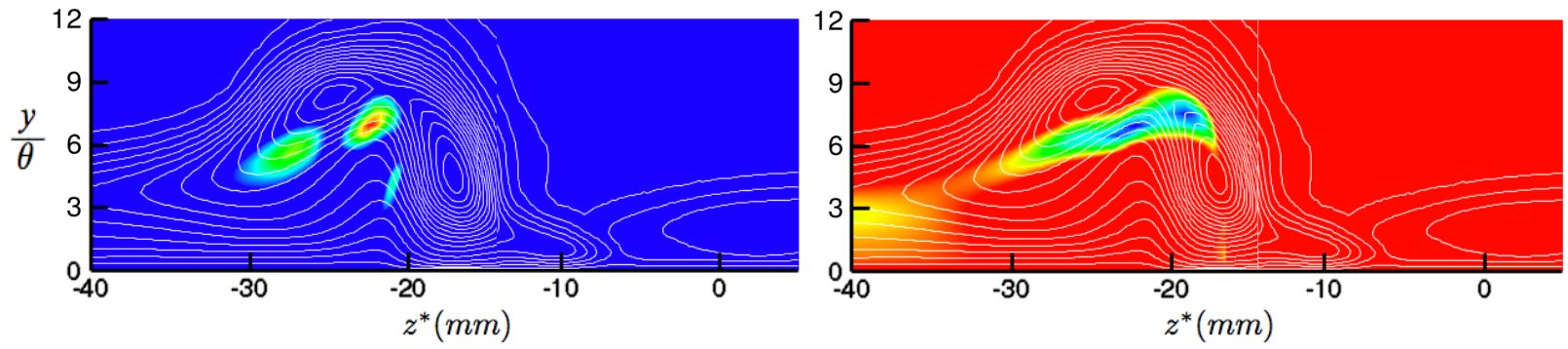

(d) $0.32 x^{*} / c$

Figure 14. Left: Instantaneous $\frac{d U}{d z}$ (Scale: $0.6-1 \frac{d U}{d z}{ }_{\text {min }}$ ) Right: Instantaneous $\frac{d U}{d y}$ (Scale: $0.6-1 \frac{d U}{d y}{ }_{\max }$ ), White lines show $u_{r m s}$ at various $x^{*} / c$ stations 


\section{Conclusion}

The capability of LES to model packets of crossflow vortices was investigated using a $45^{\circ}$ swept wing. An experiment from Chernoray et al. ${ }^{1}$ was used as the base case and results from two sub-grid models (WALE and Smagorinsky) were compared for two grids (initial and fine). As the upper airfoil surface was used for measurements in the experiment the computational model was simplified by extracting the stagnation streamline upstream of the leading edge and the streamline downstream of the trailing edge. The laminar base flow showed a typical Falkner-Skan-Cooke 3-dimensional boundary layer. When sub-grid models were compared it was found that the WALE model performed much better in the near wall region. This was expected due to the WALE model inherent ability to produce lower sub-grid scale viscosity in the wall region by including a rotational quantity in the model formulation. The WALE model was subsequently used for analysis of the stationary crossflow vortex packet.

A stationary vortex packet was generated by strong continuous suction on the surface of the airfoil for both grids. Both grids captured a packet of crossflow vortices either side of the suction hole with secondary instabilities and breakdown occurring on the left of the suction hole for both cases. It was evident that the vortices with rotation in line with the sweep induced spanwise velocity (left side) were more unstable than the vortices with opposite rotation (right side). The initial grid, however, failed to correctly model the growth and interaction between crossflow modes, and breakdown to turbulence occurred much closer than expected. The fine grid performed better in modelling the saturation of the crossflow modes and natural secondary instabilities were predicted in a similar region as in the experiment. Results showed that the ' $\mathrm{z}$ ' mode secondary instability was dominant aft of the inflection of the spanwise velocity component, which was also reported in Chernoray et al. ${ }^{1}$

The results show that LES is capable of simulating the correct growth and saturation of a packet of stationary crossflow vortices. The results were generated at a fraction of the cost of a DNS simulation and while the grid requirements remain extensive, results can be obtained using LES in the study of crossflow vortices at more realistic, higher Reynolds number, flow conditions.

\section{Acknowledgements}

The author would like to thank the Flight Physics department at Airbus, Filton for their provision of supercomputing time using the ASRC facilities \& financial support for the project. I would also like to thank Valery G. Chernoray for supplying the geometry and feedback on results.

\section{References}

\footnotetext{
${ }^{1}$ Chernoray, V. G., Doval, A. V., Kozlov, V. V., and Löfdahl, L., "Experiments on secondary instability of streamwise vortices in a swept-wing boundary layer," Journal of Fluid Mechanics, Vol. 534, June 2005, pp. 295-325.

2 Airbus, "Airbus Global Market Survey 2011-2030," Sept. 2011.

${ }^{3}$ ACARE, "European Aeronautics: A vision for 2020," July 2001.

${ }^{4}$ High level group on aviation research, "Flightpath 2050: Europe's vision for Aviation," July 2011.

${ }^{5}$ Schrauf, G., "Status and perspectives of laminar flow," Aeronautical Journal, Vol. 109, No. 1102, 2005, pp. 639-644.

${ }^{6}$ Pfenninger, W., "Some observations of the transition process on the windward face of a long yawed cylinder," Recent Developments in Boundary Layer Research, Vol. AGARDograf 97, 1965.

${ }^{7}$ Poll, D., "Some Observations of the Transition Process on the Windward Face of a Long Yawed Cylinder," Journal of Fluid Mechanics, Vol. 150, 1985, pp. 329-356.

${ }^{8}$ Saric, W. S., Reed, H. L., and White, E. B., "Stability and Transition of Three-Dimensional Boundary Layers," Annual Review of Fluid Mechanics, Vol. 35, No. 1, Jan. 2003, pp. 413-440.

${ }^{9}$ Bippes, H., "Environmental conditions and transition prediction in 3-D boundary layers," AIAA 97-1906, 1997.

${ }^{10}$ Wasserman, P. and Kloker, M., "Transition mechanism in a 3D boundary layer with pressure gradient changeover," Journal of Fluid Mechanics, Vol. 530, 2005, pp. 265.

${ }^{11}$ Malik, M. R., Li, F., Choudhari, M. M., and Chang, C.-L., "Secondary instability of crossflow vortices and swept-wing boundary-layer transition," Journal of Fluid Mechanics, Vol. 399, 1999, pp. 85-115.

${ }^{12}$ Wasserman, P. and Kloker, M., "Transition mechanisms induced by travelling crossflow vortices in a three-dimensional boundary layer," Journal of Fluid Mechanics, Vol. 483, 2003, pp. 67-89.

${ }^{13}$ White, E. B. and Saric, W. S., "Secondary instability of crossflow vortices," Journal of Fluid Mechanics, Vol. 525, 1999, pp. 275-308.

${ }^{14}$ Kloker, M., "Advanced laminar flow control on a swept wing - useful crossflow vortices and suction," 38th Fluid Dynamics Conference and Exhibit, 23-26 June, Seattle, Washington, 2008.
} 
${ }^{15}$ Schlatter, P., Large Eddy Simulation of Transition and Turbulence in Wall Bounded Shear Flow, Ph.D. thesis, Swiss Federal Institute of Technology Zurich, April 2005.

${ }^{16}$ Huai, X., Joslin, R., and Piomelli, U., "Large-eddy simulation of boundary-layer transition on a swept wedge," Journal of Fluid Mechanics, Vol. 381, 1999, pp. 357-380.

${ }^{17}$ Germano, M. P., U. Moin, P., and Cabot, W. H., "A dynamic subgrid-scale eddy-viscosity model," Physics of Fluids A: Fluid Dynamics, Vol. 3, No. 1760, 1991.

${ }^{18}$ Salman, H., Jiang, D., McGuirk, J. J., and Page, G. J., "Linear and non-linear turbulence model predictions of vortical flows in lobed mixers," Aeronautical Journal, , No. 108, 2004, pp. 65-73.

${ }^{19}$ Page, G. J., Li, Q., and McGuirk, J. J., "LES of Impinging Jet Flows Relevant to Vertical Landing Aircraft," The 23rd Applied Aerodynamics Conference, Toronto, Canada, 2005.

${ }^{20}$ Rife, R. E. and Page, G. J., "Large Eddy Simulation of high Reynolds number jets with microject injection," 17th AIAA/CEAS Aeroacoustics Conference, Portland, Oregon, 2011.

${ }^{21}$ Pokora, C. D., McMullan, W., Page, G. J., and McGuirk, J. J., "Influence of a numerical boundary layer trips within LES of a subsonic jet on spatio-temporal correlations," 17th AIAA/CEAS Aeroacoustics Conference, Portland, Oregon, 2011.

${ }^{22}$ Smagorinsky, J., "General circulation experiments with the primitive equations I. The basic experiment," Monthly Weather Review, Vol. 91, 1963, pp. 99-164.

${ }^{23}$ Van Driest, E. R., "On turbulent flow near a wall," Journal of the Aeronautical Sciences, Vol. 23, 1956, pp. $1007-1011$.

${ }^{24}$ Nicoud, F. and Ducros, F., "Subgrid-Scale Stress Modelling Based on the Square of the Velocity Gradient Tensor," Flow, Turbulence and Combustion, Vol. 62, 1999, pp. 183-200.

${ }^{25}$ Piomelli, U. and Balaras, E., "Wall-layer models for large-eddy simulations," Annual Review Of Fluid Mechanics, Vol. 34, 2002, pp. 349-374.

${ }^{26}$ Jeong, E. and Hussain, F., "On the identification of a vortex," Journal of Fluid Mechanics, Vol. 285, 1995, pp. 69-94. 